\title{
CT imaging features of carotid artery plaque vulnerability
}

\author{
Alessandro Murgia ${ }^{1}$, Marco Erta $^{1}$, Jasjit S. Suri ${ }^{2}$, Ajay Gupta ${ }^{3}$, Max Wintermark $^{4}$, Luca Saba ${ }^{1}$ \\ ${ }^{1}$ Department of Radiology, Azienda Ospedaliero Universitaria (A.O.U.), di Cagliari - Polo di Monserrato, s.s. 554 Monserrato (Cagliari), Italy; \\ ${ }^{2}$ Stroke Monitoring and Diagnosis Division, AtheroPoint(tm), Roseville, CA, USA; ${ }^{3}$ Department of Radiology, Weill Cornell University, New York, \\ NY, USA; ${ }^{4}$ Department of Radiology, Stanford University, Stanford, CA, USA \\ Contributions: (I) Conception and design: A Murgia, L Saba; (II) Administrative support: A Murgia, L Saba; (III) Provision of study materials or \\ patients: A Murgia, L Saba; (IV) Collection and assembly of data: A Murgia, JS Suri, L Saba; (V) Data analysis and interpretation: A Murgia, JS Suri, \\ L Saba; (VI) Manuscript writing: All authors; (VII) Final approval of manuscript: All authors. \\ Correspondence to: Luca Saba, MD. Department of Radiology, Azienda Ospedaliero Universitaria (A.O.U.), di Cagliari - Polo di Monserrato s.s. 554 \\ Monserrato (Cagliari) 09045, Italy. Email: lucasaba@tiscali.it.
}

\begin{abstract}
Despite steady advances in medical care, cardiovascular disease remains one of the main causes of death and long-term morbidity worldwide. Up to $30 \%$ of strokes are associated with the presence of carotid atherosclerotic plaques. While the degree of stenosis has long been recognized as the main guiding factor in risk stratification and therapeutical decisions, recent evidence suggests that features of unstable, or 'vulnerable', plaques offer better prognostication capabilities. This paradigmatic shift has motivated researchers to explore the potentialities of non-invasive diagnostic tools to image not only the lumen, but also the vascular wall and the structural characteristics of the plaque. The present review will offer a panoramic on the imaging modalities currently available to characterize carotid atherosclerotic plaques and, in particular, it will focus on the increasingly important role covered by multidetector computed tomographic angiography.
\end{abstract}

Keywords: Carotid; atherosclerosis; computed tomography angiography (CTA); vulnerable plaque

Submitted Jan 21, 2020. Accepted for publication Apr 24, 2020.

doi: $10.21037 /$ atm-2020-cass-13

View this article at: http://dx.doi.org/10.21037/atm-2020-cass-13

\section{Cardiovascular disease}

\section{The numbers of atherosclerosis}

Atherosclerosis (ATS) is a chronic vascular disease which represents one of the major causes of morbidity and mortality worldwide. A number of modifiable risk factors for atherosclerosis have been described, such as smoking, dyslipidemia, high blood pressure, hypercholesterolemia, and diabetes mellitus, among others.

The first sign of ATS appears to be the presence of fatty streaks in arterial walls, which progressively develop into the so-called atheroma and vascular plaques. The clinical consequences of these plaques vary according to their location, the degree of resulting stenosis and their growth rate (1).

In particular, for what concerns carotid plaques, even though certain types of atheromata can remain stable for many years, others rupture (unstable or vulnerable plaques). Such event can lead to local thrombus formation and subsequent embolisation to the ipsilateral ophthalmic, middle cerebral, or anterior cerebral arteries. As a result, a number of clinical manifestations may ensue, such as ipsilateral amaurosis fugax or retinal infarction, controlateral body transient ischaemic attack (TIA) and stroke (2).

Since atherosclerosis is most frequently asymptomatic, it is difficult to determine its incidence with accuracy. However, coronary heart disease appears to be the leading cause of death in Western countries, with more than 370,000 deaths per year. A population study designed to investigate the prevalence of asymptomatic carotid atherosclerotis in the general population showed that its general prevalence could be as high as $25 \%$ in adults $(3,4)$. 


\section{Pathogenesis of the atherosclerotic plaque}

The vast majority of strokes are of ischemic etiology and up to $30 \%$ correlate with the presence of carotid atherosclerotic plaques $(5,6)$.

The etiopathogenesis of the atherosclerotic plaque is multifaceted. There is general agreement that atherosclerosis stems from low density lipoproteins (LDLs) accumulating in the arterial intima, where they are prone to undergo oxidative processes that stimulate the immune response (7).

As a result, endothelial cells and smooth muscle cells (SMCs) express adhesion molecules and other chemical mediators which, in turn, facilitate the transformation of monocytes into macrophages and, through the accumulation of intracellular lipid content, foam cells, which stack in several layers giving rise to the visible yellow-colored xanthomas or fatty streaks.

Smaller lipid pools deposit beneath the layers of foam cells determining intimal thickening, while larger and more confluent lipid clusters attract the invasion of macrophages and grow into necrotic cores (also named lipid-rich necrotic core). In the presence of a necrotic core, the lesion is named a fibroatheroma.

The loose fibrocellular tissue which forms the atherosclerotic lesion often proliferates and intervenes between the necrotic core and the luminal surface, forming the so-called fibrous cap. Likewise, the necrotic core can progressively calcify, sometimes occupying most of the plaque volume.

Subsequently, vasa vasorum grow into the atherosclerotic lesions; these plaque neovessels are typically fragile and permeable, predisposing to intraplaque bleedings. Alternatively, plaque hemorrhage may result from a ruptured fibrous cap (8).

During atherogenesis, mechanisms of remodeling take place; in particular, in expansive remodeling the lumen area is partially preserved until plaques are large, while in constrictive remodeling stenosis may result from progressive plaque growth or vascular bed shrinkage. While the two mechanisms often coexist, it is interesting to note that expansive remodeling typically occurs with fibroatheromas and positively correlates with plaque inflammation, medial atrophy, and necrotic core size; on the contrary, constrictive remodelling often contain lesions rich in fibrous tissue (9).

Plaque rupture/erosion is the most frequent cause of thrombosis. Through a structural defect in the fibrous cap, especially where the cap is thin and infiltrated by foam cells (thin-cap fibroatheromas; TCFAs), the highly thrombogenic core contacts the blood with the formation of a thrombus.

To characterize the severity and prognosis of plaques, several terms are used.

Plaque burden is a measure of the extent of atherosclerosis. It can be measured through a variety of descriptive methods, such as: plaque volume, extent of lesion along the arterial surface, coronary calcium score by Computed tomography (CT), intima-media thickness and plaque area by ultrasound (US) and ankle-brachial pressure index in peripheral vascular disease.

Plaque vulnerability describes the short-term risk of thrombosis. A number of histological features of ruptureprone plaques have been identified, such as: a TCFA with a large necrotic core and macrophage infiltration in the cap, big plaque size, expansive remodeling, neovascularization, intraplaque hemorrhage (IPH), adventitial inflammation and spotty calcifications (10).

The American Heart Association (AHA) proposed a plaque classification that identifies six types which are meant to reflect the temporal natural history of the disease. Type I: atherogenic lipoproteins and mononuclear infiltrates; medial thickening. Type II: fatty streaks. Type III: lipid particles disrupt the integrity of the SMCs. Type IV: typical atheromata (large extracellular lipid core). Type $\mathrm{V}$ : atheromata developing fibrous caps. Type $\mathrm{Vb}$ : largely calcified lesions. Type Vc: more fibrous connective tissue, little lipid and no calcium. Type VI: ruptured atherosclerotic plaque with IPH or thrombosis (11-13). The rationale for this classification is that the structural composition of the carotid plaque modifies with the progression of the disease and some phases appear to be more prone to acute vascular events than others; in fact, lesion types IV, V and VI are those typically involved in cerebrovascular events.

\section{A change of perspective: from measuring stenosis to identifying vulnerability}

For several years the degree of luminal stenosis had been considered the main factor affecting the severity of carotid atherosclerotic disease.

Indeed, a number of trials published along the course of the 1980s and 1990s, such as the European Carotid Surgery Trial (ECST), the North American Symptomatic Carotid Endarterectomy Trial (NASCET) and the Asymptomatic Carotid Atherosclerosis Study (ACAS), contributed to establishing the idea that the degree of luminal stenosis should be the reference point to indicate 

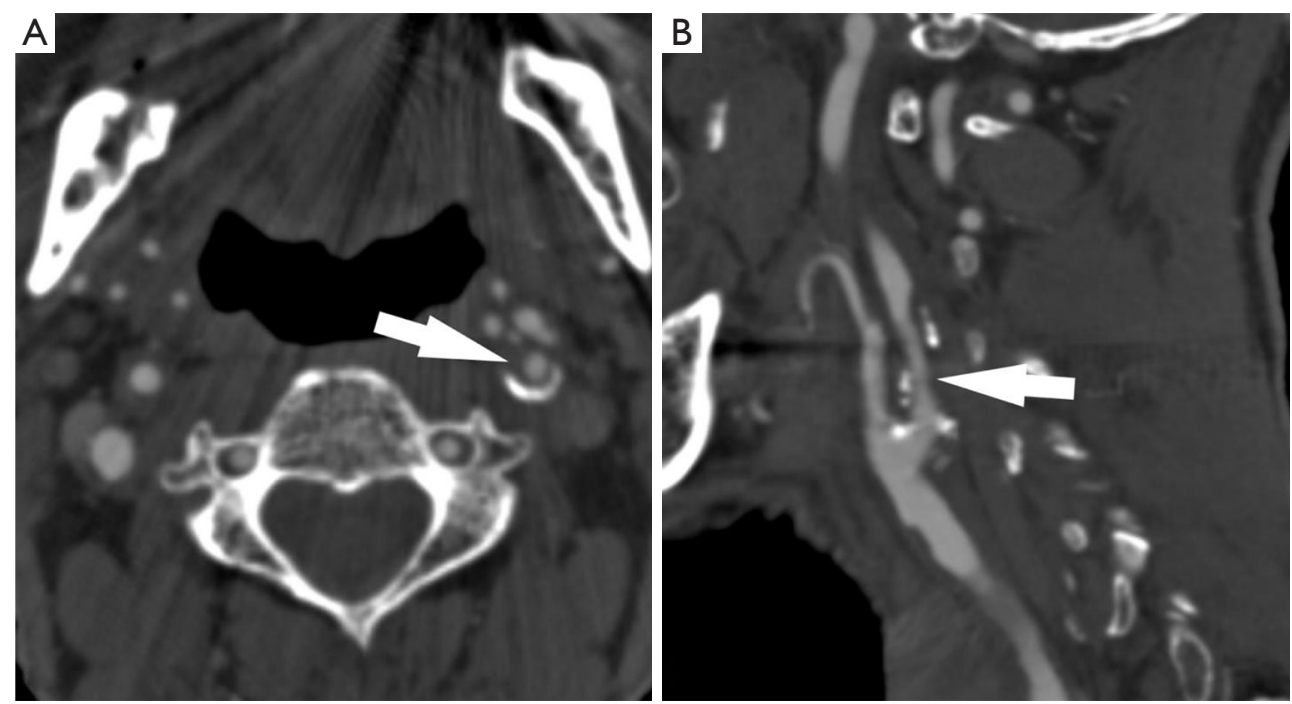

Figure 1 A 73-year-old male with TIA. In the left ICA a mixed plaque is visible (white arrow) which determines a 50\% NASCET degree of stenosis (A: axial scan, B: sagittal plane). Calcifications can be seen in the outer part of the plaque with the "eggshell" type configuration.

the appropriateness of carotid endarterectomy intervention (14-17) (Figure 1).

More recently, a growing body of scientific evidence suggests that plaque structure and composition may serve as a more reliable biomarker for the prediction of cerebrovascular ischemic events (18).

Morphological high risk features, such as intima-media thickness, lipid cores, thin fibrous caps and ulcerated plaques have shown high correlation with stroke, even in patients with mild to moderate stenosis. Conversely, high calcium content appears to correlate with more stable plaques $(12,19)$.

Thus, while it is generally accepted that high-grade stenosis tends to associate with complicated plaques more frequently than nonstenotic asymptomatic lesions, a large number of scientific publications demonstrated that complicated plaques also occur with moderate stenosis. As a result, nowadays the recognition of high risk carotid artery lesions can no longer be based on the degree of stenosis alone (20).

In a cross-sectional observational study including 85 consecutive patients with anterior circulation embolic stroke of undetermined source, an association was observed with large but nonstenotic carotid artery plaques located ipsilateral to the brain lesion, rather than controlateral; the Authors suggest that nonstenotic plaque is an underestimated cause of stroke (21).

As part of a substudy of the AIM-HIGH (Atherothrombosis
Intervention in Metabolic Syndrome with Low HDL/ High Triglycerides: Impact on Global Health Outcomes) clinical trial, an investigation was carried out to evaluate whether carotid plaque features of vulnerability may serve as a marker of cardiovascular outcomes in patients with a known history of atherosclerotic disease. Results showed that lipid content and fibrous cap integrity strongly predicted systemic atherothrombotic risk (22).

A retrospective study, conducted in a matched cohort of Caucasian and East Asians patients with stable chest pain, aimed to investigate the reasons behind the lower prevalence of myocardial infarction in the East Asian population. Coronary atherosclerotic burden and plaque composition were compared via computed tomography angiography for the two subgroups; results showed that that east Asians appear to have a lower predominance of high risk noncalcified plaques (23).

\section{Imaging of the carotid atherosclerotic plaque}

\section{What's in the toolbox?}

Up until carotid atherosclerosis risk was evaluated based on the degree of stenosis alone, diagnosis and classification of carotid plaques was possible only in advanced stages of the disease, when luminal stenosis became manifest through digital subtraction angiography (DSA) or organ perfusion techniques. Then, DSA represented the diagnostic gold standard thanks to its capability not only to measure 
stenosis, but also to predict plaque rupture by identifying ulceration and surface abnormalities (12).

However, thanks to recent advances in medical imaging, new opportunities emerged to explore and assess both lumen morphology and the lumen wall itself. Similarly, the shift of attention which took place in this field of literature away from stenosis and into aspects of vulnerability has spurred tremendous interest for the development of noninvasive techniques to identify the characterizing features of vulnerable plaques (24).

Before focusing with more detail into CT imaging features of vulnerable plaques, the present section will provide a panoramic view of the various diagnostic tools currently available.

\section{Ultrasound (US)}

US represents the first-line imaging modality in the evaluation of carotid artery plaques since it is relatively simple, inexpensive and widely available.

The measurement of intima media thickness (IMT) has been widely recognized as a marker of systemic atherosclerosis with a strong correlation to coronary artery disease $(25,26)$. However, a number of recent studies indicate for IMT a poor prognostic value and, at present, the use of IMT to guide therapeutical decisions is not recommended (27). Besides, it has been observed that carotid atherosclerosis is focal and manifests with plaques while IMT is often measured deliberately in areas not affected by plaque formation; indeed, IMT and plaques are biologically and genetically distinct entities which represent different phenotypes of atherosclerosis and respond differentially to therapy (28).

As far as B-mode images are concerned, high lipid content and intraplaque haemorrhage appear echolucent, while calcium and fibrous tissue appear echogenic (29). However, most Authors agree that US cannot reliably discriminate lipid from IPH because of a substantial overlap in their echo-structure (30). Moreover, US is heavily limited by being a user-dependent methodology.

Contrast-enhanced ultrasound is an emerging technique that finds its rationale in the selective retention of contrast at specific sites of disease. There is evidence that plaque enhancement in contrast-enhanced ultrasound correlates with greater neovascularization at histology (31-34).

Moreover, current research is testing targeted microbubbles (attached to antibodies, small peptides, and glycoproteins such as VCAM-1 and P-selectin) designed to identify specific cell surface structures (35). However, molecular US imaging is limited by the fact that it can only assess intravascular events beyond a given threshold of molecular expression.

Recent research has tested the potentialities of 3D US in the evaluation of plaque burden. In particular, within the context of PESA (Progression of Early Subclinical Atherosclerosis) observational prospective cohort study, 4,184 volunteers (aged between 40 and 54 years and without a history of cardiovascular disease) underwent carotid and femoral 3-dimensional vascular ultrasound (3DVUS), with a follow up at 3 and 6 years. The Authors employed an automated 30-degree sweep capturing approximately $6 \mathrm{~cm}$ of arterial length, centered at the carotid bulb and at the femoral bifurcation. Results showed that measures of plaque burden correlate with common cardiovascular risk factors with a higher prognostic value than plaque detection alone (36). While this study gave significant contribution towards the definition of a reliable imaging method to appraise plaque burden, further research is needed in order to develop optimal semi-automated scanning systems, test the feasibility to include femoral plaque measurement (in addition to carotid measurement) in the clinical routine and establish whether the evaluation of a limited arterial segment can provide a comprehensive assessment of plaque burden (37).

\section{Magnetic resonance imaging (MRI)}

High-resolution MRI has been widely used to evaluate atherosclerotic plaques with a particular ability to characterize soft tissue components. It can identify with high accuracy, sensitivity and specificity the lipid rich necrotic core, thin and ruptured caps, IPH (38).

Serfaty et al. published an AHA classification of atherosclerotic plaque specifically adapted for MR imaging, highlighting the ability of MR to describe plaques on the basis of composition and morphology (39).

One of the earliest applications of nanotechnology in MRI exploited the phagocytic uptake of paramagnetic iron oxide particles by macrophages at the plaque level (40). More recently, target-specific contrast agents (bound to adhesion molecules, high-density lipoprotein, MMPs, macrophage scavenger receptors and fibrin) have been developed, which allow for a better representation of plaque components and reduce acquisition times.

However, MRI is signal-insensitive and has low spatial and temporal resolution, which translates in long scanning times. Moreover, it is complex, expensive and less readily available when compared to other imaging modalities. 


\section{Multi detector computed tomography angiography (MDCTA)}

Recently, there has been growing interest in MDCTA as a non-invasive modality to characterize atherosclerotic plaques. When compared with MRI, CT is more signalefficient and has higher spatial and temporal resolution.

MDCTA can also detect various important features associated with plaque vulnerability (41), which will be further analyzed in the next section.

\section{Positron emission tomography (PET)}

PET with F-18-fluorodeoxyglucose (FDG) is a molecular imaging technique that can illuminate metabolically active processes. It has been shown to detect 'inflamed plaques' by highlighting FDG uptake in macrophages at the plaque level.

In a cohort of 54 patients scheduled for carotid endarterectomy, Cocker et al. demonstrated a correlation between ${ }^{18} \mathrm{FDG}$ uptake and the presence of markers of intraplaque inflammation at the immunohistologic analysis of the surgical specimen (such as macrophagesensitive CD68 and leukocyte-sensitive CD45); moreover, results showed a greater degree of ${ }^{18} \mathrm{FDG}$ uptake in symptomatic patients, especially those with a recent history of cerebrovascular events ( $<90$ days), suggesting that ${ }^{18}$ FDG imaging with PET/CT may serve as a surrogate biomarker of plaque activity and allow for non-invasive interrogation of the inflammatory status of plaque (42). Similarly, a number of publications explored the capability of ${ }^{18} \mathrm{~F}$-sodium fluoride PET to distinguish vulnerable from non-vulnerable plaques with promising results, with the potential to detect early stages of atherosclerotic development (43). Indeed, while macroscopic calcium seems to stabilize atherosclerotic plaques, microcalcific deposits appear to increase the risk of plaque rupture by amplifying mechanical stress at the level of fibrous cap. It is postulated that ${ }^{18} \mathrm{~F}$-sodium fluoride replaces the hydroxyl groups of hydroxyapatite expressed in regions of active mineralization; hence, elevated ${ }^{18} \mathrm{~F}$-sodium fluoride uptake in PET/CT imaging could suggest increased risk of mechanical failure, which translates in plaque instability (44). However, for the time being molecular imaging is less widely available and requires longer procedural times than other imaging options such as CT or MRI.

\section{Immunoscintigraphy}

Immunoscintigraphy uses radioactive tracers (such as low-density lipoproteins, haematoporphyrin derivatives, fibronectin, immunoglobulins, platelets and antifibrin antibodies) that localize into vulnerable plaques.

\section{A look into the future}

Shear wave elastography (SWE) is a novel US method which could identify plaques prone to rupture measuring acoustic radiation propagation within the fibrous cap (45).

A number of serum biomarkers (mainly inflammatory and proteolytic markers such as: high-sensitivity C-reactive protein, IL-6, MMP-9, MMP-2, and tissue inhibitors of metalloproteinases) have shown to be highly predictive of plaque vulnerability (46).

Optical coherence tomography (OCT) is an invasive imaging technique which is capable of visualizing subsurface tissue in high detail (with a spatial resolution of $10 \mu \mathrm{m}$ ), demonstrating lipid content, fibrous cap thickness, and fibrous cap macrophage attenuation. So far, OCT has been employed in the study of the coronary artery bed, but preliminary research indicates it could also find application in the evaluation of carotid plaques (12).

Artificial intelligence could play a fundamental role in the future; in particular, deep learning might enable automated detection of quantitative biomarkers and provide risk stratification protocols that incorporate imaging features from different techniques $(47,48)$.

\section{CT imaging of the vulnerable plaque}

\section{Features of vulnerability}

The use of CT in the evaluation of carotid plaques has received steadily growing attention in recent years, especially since the advent of multi detector CT scanners (MDCT), which enable fast and accurate acquisition of vascular structures with minimal discomfort to the patient. Additionally, CTA allows in the same session to evaluate extra- and intracranial vessels (from the aortic arch to the circle of Willis) and cerebral parenchymal abnormalities. CTA has been shown to have sensitivity and specificity comparable to those of DSA in the detection of carotid artery stenosis and plaque surface irregularities, but it can also show the vascular wall and provide significant information on plaque volume and composition (49). The present section will provide an overview of the main imaging biomarkers of vulnerable carotid plaques as they appear in MDCTA. 


\section{Intraplaque haemorrhage}

There is general agreement among Authors that IPH can be considered the most relevant imaging feature associated with plaque instability (50-54). It has also been demonstrated that IPH prevalently locates ipsilateral to embolic strokes of undetermined source (52).

In the past years, a significant number of publications have indicated MRI as the best imaging modality to detect IPH based on the oxidative state of haemoglobin, which can be highlighted using common imaging sequences (47). Besides, many Authors argue that CT is insensitive in the differentiation of fibrous, lipid, and intraplaque haemorrhage because of a substantial overlap in HU values for these tissues.

However, a number of recent publications have been challenging this concept, indicating that modern MDCTA, paired with dedicated segmentation software analysis, can provide equal or better results in the detection of soft tissue subcomponents of the plaque when compared to MRI.

In this regard, in a retrospective study by Saba $e t$ al. conducted on 91 patients who underwent CEA, CTA carotid plaque slices were matched and compared to the corresponding histologic section to determine the accuracy of CTA in the detection of IPH. Results showed that IPH is characterized by low average values of HU and that a threshold of $25 \mathrm{HU}$ in the volume acquired after the administration of contrast medium provides excellent sensitivity and specificity for IPH (53).

Wintermark et al. conducted a study to compare CTA-derived plaque sections, analyzed via an automated classification computer algorithm, versus histologic corresponding slices as the gold standard. The authors observe that while much of the literature indicates MRI and US as the methodologies of choice to study the composition of carotid plaques, most publications on CT have been based on older-generation scanners and that the potential for modern MDCTA in this field is unexploited. Their study shows that the CT-derived algorithm provide excellent correlation with histologic examinations in the quantification of calcifications; lipid cores and IPH also show good correlation, although with some limitations when smaller areas of lesion are considered (51).

Another retrospective study by Saba $e t$ al., which included 123 subjects (246 carotid arteries), quantified the degree of stenosis and plaque composition using CTA. Subcomponent volumes of the plaque were analyzed via a dedicated semiautomatic segmentation software which uses an algorithm of iterative image restoration and subdivides tissue components into HU threshold classes. Expanding from the classic definition of three plaque types based on attenuation values described by de Weert et al. (lipid, mixed, calcified) (55), five classes were identified: calcium $(>130 \mathrm{HU})$, fibrous tissue (60 to $130 \mathrm{HU}$ ), lipid tissues ( $<60 \mathrm{HU}$ ), lipid-IPH (26 to $59 \mathrm{HU})$, IPH (<25 HU). Relative percentages for each component were also calculated, including a ratio of IPH/ lipid volume ranging from value 0 (no IPH) to value 1 (all the lipid plaque correspondent to IPH). Results showed a statistically significant difference for the lipid and, in particular, for the IPH volumes across the symptomatic and asymptomatic patient groups, confirming that IPH greater volumes correlate with symptoms and cerebrovascular events. Conversely, in asymptomatic subjects a higher relative percentage of calcium and lipid-IPH was found. The study demonstrated that it is not only the overall plaque volume to be predictive of ruptures, but also the relative percentage of plaque subcomponents, suggesting that the biomechanical characteristics of the plaque are involved in the triggering of acute cerebrovascular events. Additionally, this study provides proof of principle that MDCTA, coupled with the use of volumetric quantification analysis software, is a reliable modality to image even small changes in plaque composition (56). Moreover, CT offers a unique alternative in those patients who have a contraindication to undergoing MRI imaging (e.g., prosthesis or pacemaker). Similar results have been demonstrated in a number of other recent works $(57,58)$.

\section{Lipid-rich necrotic core (LRNC)}

The presence of a LRNC has been associated with increased risk of stroke (59).

In a similar fashion to what has been earlier discussed with regards to IPH, many publications over the course of the years have contributed to the idea that CT is a low performer in the identification of LRNC, due to a substantial overlap in HU with IPH (both showing values $<60 \mathrm{HU})$. However, a number of Authors bring forward the idea that recent technological advances in MDCTA and volumetric analysis software allow room for a redefinition of the role of CTA in this diagnostic field.

In particular, Saba et al. compared the accuracy of in vivo quantification of calcification, LRNC and matrix from CTA sections against the histological specimens of 31 endarterectomies (with a total of 239 cross sections) by using dedicated image processing algorithms. The Authors start from the assumption that MRI does not offer signal intensity values characteristic of the different 
soft tissue plaque components within a single sequence, making it necessary to perform a differential analysis across various sequences, resulting in complex and timeconsuming protocols. Additionally, results published in most works adopt a dedicated neck coil to compensate for insufficient spatial resolution. Conversely, HU values in CTA have the potential to be more quantitative than MRI signal intensity. Results from this work showed high correlation with histologic specimens in the quantification of calcification, demonstrating that the overestimation of calcified plaques at CT angiography, often described and classically attributed the artifactual "blooming effect", may be successfully overcome via the use of dedicated software algorithms. Similarly, the software demonstrated a low level of measurement bias for what concerns the quantification of LRNC. Therefore, the Authors conclude that algorithm approach not only enables an accurate quantification of calcification, but also provides satisfactory characterization of soft plaque subregions (60).

\section{Thin fibrous cap (TFC)}

There is evidence based on histological findings that an intact fibrous cap correlates with low risk of plaque rupture, whereas a TFC and fissured fibrous cap (FFC) are associated, respectively, with moderate and high risk. Thus, the typical unstable plaques appear as TFC covering a LRNC, which can be accurately documented via MRI $(61,62)$ and CTA $(63)$.

In particular, it was demonstrated with histological validation that in CTA the presence of FFC correlates with a higher degree of plaque enhancement when compared against non-fissured caps (64).

\section{Neovascularization}

Inflammatory processes, often referred to by the term 'plaque activity', play a central role in plaque progression, with intraplaque neovascularisation being one of its typical signs and a prelude for neovessel rupture and haemorrhage (65).

There is evidence that CTA can detect and quantify intraplaque neovascularisation, which appears to be proportional to the amount of contrast enhancement (66).

\section{Plaque thickness}

Evidence shows that maximum plaque thickness, a parameter accurately measurable in CT which reflects plaque volume, correlates with the incidence of ischaemic symptoms and stroke $(67,68)$.

A recent study demonstrated, in fifty-one patients admitted for ischemic stroke or transient ischemic attack (TIA), a strong correlation between maximum soft plaque thickness (assessed on CTA) and American Heart Association type VI plaques (previously defined by MRI) (69).

In a cross-sectional study, Gupta et al. retrospectively analyzed a series of carotid CTA performed in patients with high-grade carotid stenosis in order to assess the correlation between plaque thickness and stroke or transient ischemic attack (TIA) history. The Authors found that, compared with asymptomatic subjects, patients with symptomatic carotid disease showed much larger soft (non-calcified) plaque and total plaque thickness values; conversely, symptomatic patients had smaller hard (calcified) plaque thickness measurements. Moreover, the study showed for each $1-\mathrm{mm}$ increase in soft plaque there was a 2.7 times greater odds of previous stroke or TIA (70).

\section{Surface morphology}

Plaque surface can be classified as smooth, irregular (cavities from 0.3 to $0.9 \mathrm{~mm}$ ) or ulcerated (cavities $>1 \mathrm{~mm}$ ) (71). In particular, ulcerations (Figure 2) are considered a marker of vulnerable plaques (72), although they may also represent relics of previously occurred ruptures (73).

de Weert et al. explored the correlation between plaque surface morphology (together with degree of stenosis and other cardiovascular risk factors) and cerebrovascular symptoms, demonstrating a higher incidence of complex plaques in symptomatic patients (74).

It has been demonstrated that MDCTA has sensitivity and specificity higher than DSA and US in the detection of ulcerations $(75,76)$. Sometimes also thrombus in the plaque can be detected (Figure 2).

\section{Plaque total volume and subcomponents}

There is scientific evidence that the volume of the carotid artery plaque associates with plaque vulnerability and the incidence of stroke (77). In an US-based study designed to evaluate plaque progression in a cohort of 349 patients attending vascular prevention clinics, Wannarong et al. demonstrated that total plaque volume can predict cardiovascular outcomes more reliably than both IMT and total plaque area (78). The good spatial resolution of CT allows for a satisfactory analysis of both total plaque volume and of its subcomponents, especially when coupled with semi-automated software analysis based on HU thresholds and image restoration algorithms. Similar segmentation protocols have been tested using MRI, but the results were less convincing due to unsatisfactory spatial resolution (79). 

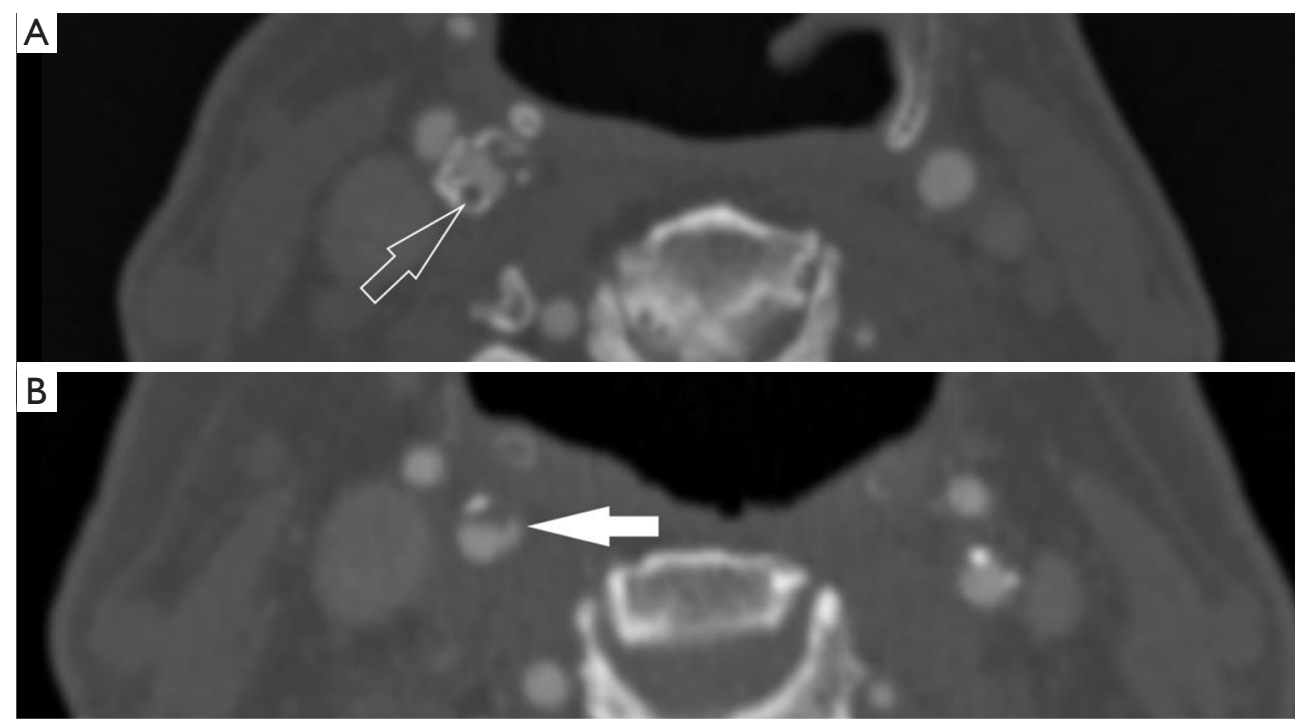

Figure 2 A 79-year-old male with left stroke. (A) In the right ICA a hypodense area is visible (white open arrow) which is due to a thrombus. (B) (5 mm above A): an ulcerated plaque is visible (white arrow).

As it was discussed earlier, while previous research indicated a higher sensitivity from MRI in discriminating between soft tissue components of the plaque, recent evidence suggests that modern MDCTA, coupled with dedicated software analysis, can discriminate accurately between lipid component and IPH (56).

With regards to the relative percentage of plaque subcomponents, as it was discussed earlier, there is evidence that fatty content associates with higher-risk lesions, while calcium appears to be a protective factor by supposedly stabilizing the plaque against biomechanical stress.

Saba et al. demonstrated an association between cerebral microbleeds, commonly seen in stroke patients and considered to be a harbinger of cerebral hemorrhage (80), and increased volume of carotid plaque fatty subcomponent. In this study, cerebral microbleeds were evaluated using a T2*-weighted gradient-recalled echo sequence, while carotid arteries were imaged in MDCTA (81).

A new type of CT scanner, dual energy CT, which works by postprocessing data relative to the attenuation values obtained from tissues scanned at multiple energy levels, has shown the ability to reduce artifacts related to the presence of calcium and quantify plaque calcifications with increased accuracy (82-84).

\section{Present technique limitations}

Despite MDCTA holding a significant potential to become the leading imaging modality for the study of carotid plaques, there are a number of technique limitations that must be highlighted.

First, as it was discussed in previous sections, there still is a certain degree of controversy in the literature with regards to the capability of CTA to reliably characterize soft tissue components, with some Authors indicating low sensitivity in the discrimination of lipid core and IPH, especially when small lesions are considered (54).

Other limitations described in the literature include beam-hardening artifacts associated with calcification (socalled ballooning artifacts, which can be partly reduced with post processing algorithms and DECT) and a high variability in patterns of plaque enhancement (which appears to be highly dependent on blood flow and time delay of image acquisition) $(56,82,85)$.

Finally, exposure to ionizing radiation, which can be partly reduced with modern scanners and DECT (86), and adverse reactions to iodine-based contrast agents (49).

\section{The role of plaque imaging in the management of cardiovascular disease}

\section{Implementing research into the everyday clinical scenario}

A growing number of recent scientific publications indicate that risk stratification in carotid atherosclerosis cannot rely on the quantification of luminal stenosis alone, but that 
newly introduced features of plaque vulnerability can be a more dependable predictor of acute vascular events. Even so, in the majority of institutions, US-based measurements of the degree of stenosis still represent the routine clinical examination to guide therapeutical approach.

In this scenario, MDCTA represents a potential candidate to become the imaging modality of choice in the characterization of carotid plaques, thanks to a number of advantages, such as: high spatial resolution, short acquisition times, large coverage in the $\mathrm{Z}$-axis, access to a number of newly emerging post-processing techniques and a relatively easy to read interface for clinicians and surgeons (18).

Moreover, MDCTA is widely available in most clinical infrastructures and it is already integrated as standard care for most patients admitted for cerebrovascular incidents (12).

\section{What do clinicians want to know?}

Fundamentally, the imaging of carotid atherosclerosis is aimed at identifying patients with high risk of future cardiovascular events (especially among asymptomatic groups), guide therapeutical approaches and improve longterm outcomes (87).

When fully implemented in the clinical routine, the identification of higher risk biomarkers may warrant closer clinical follow-up and, possibly, expedite intensive medical treatment or revascularization in selected patient (47).

Furthermore, imaging provides support to the ongoing research in the field of new medical treatment. Indeed, imaging can provide objective assessment of a drug's clinical efficacy before the ultimate step in drug evaluation (phase 3 trials, designed to assess impact on clinical end points) is initiated, involving the enrolment of large cohorts of patients, long follow-up periods and, typically, enormous costs. In other words, atherosclerosis imaging allows for the monitoring of early signals of therapeutical effects, which can inform researchers on which drugs hold a higher chance for success and merit to progress into phase 3 evaluation stages.

In particular, MDCTA has been used to monitor the effects of statins, showing modifications of plaque composition over time, with a progressive reduction of fatty subcomponents $(88,89)$.

Additionally, it has been demonstrated that the identification of high risk markers of carotid atherosclerosis in MDCTA can predict 10-year atherosclerotic cardiovascular disease risk scores $(57,90)$.

\section{Cost-effectiveness}

There is evidence that institutions that plan therapeutical approaches to cardiovascular disease by integrating clinical information with imaging biomarkers experience benefits in terms of cost-effectiveness $(47,91)$.

Moreover, it has been demonstrated that CTA and MRI both prove more cost-effective than DSA (92); Sheahan et al. indicate that MRI appears to be more costly and timeconsuming in the clinical routine when compared against MDCTA (60).

\section{Conclusions}

While scientific evidence suggests that imaging features of carotid plaque vulnerability outperform measurements of degree of stenosis in cerebrovascular risk stratification capabilities, a discrepancy still exists with the everyday clinical scenario since in most institutions such techniques are yet to find implementation in the clinical routine. In this context, CTA represents a strong candidate to become the imaging modality of choice in carotid plaque characterization, thanks to its excellent spatial resolution, short acquisition times, widespread availability and the capacity to accurately define plaque volume and subcomponents, especially when coupled with postprocessing analysis techniques.

\section{Acknowledgments}

Funding: None.

\section{Footnote}

Provenance and Peer Review: This article was commissioned by the Guest Editor (Dr. Kosmas I. Paraskevas) for the series "Carotid Artery Stenosis and Stroke: Prevention and Treatment Part I" published in Annals of Translational Medicine. The article was sent for external peer review organized by the Guest Editor and the editorial office.

Conflicts of Interest: All authors have completed the ICMJE uniform disclosure form (available at http://dx.doi. org/10.21037/atm-2020-cass-13). The series "Carotid Artery Stenosis and Stroke: Prevention and Treatment Part I" was commissioned by the editorial office without any funding or sponsorship. The authors have no other conflicts 
of interest to declare.

Ethical Statement: The authors are accountable for all aspects of the work in ensuring that questions related to the accuracy or integrity of any part of the work are appropriately investigated and resolved.

Open Access Statement: This is an Open Access article distributed in accordance with the Creative Commons Attribution-NonCommercial-NoDerivs 4.0 International License (CC BY-NC-ND 4.0), which permits the noncommercial replication and distribution of the article with the strict proviso that no changes or edits are made and the original work is properly cited (including links to both the formal publication through the relevant DOI and the license). See: https://creativecommons.org/licenses/by-nc-nd/4.0/.

\section{References}

1. Herrington W, Lacey B, Sherliker P, et al. Epidemiology of Atherosclerosis and the Potential to Reduce the Global Burden of Atherothrombotic Disease. Circ Res 2016;118:535-46.

2. Thapar A, Jenkins IH, Mehta A, et al. Diagnosis and management of carotid atherosclerosis. BMJ 2013;18;346:f1485.

3. Prati P, Vanuzzo D, Casaroli M, et al. Prevalence and determinants of carotid atherosclerosis in a general population. Stroke 1992;23:1705-11.

4. Sturlaugsdottir R, Aspelund T, Bjornsdottir G, et al. Prevalence and determinants of carotid plaque in the cross-sectional REFINE-Reykjavik study. BMJ Open 2016;24;6:e012457.

5. Caplan LR. Diagnosis and treatment of ischemic stroke. JAMA 1991;266:2413-18.

6. Warlow C, Dphil C, Dennis M, et al. Stroke. Lancet 2003;362:1211-24.

7. Falk E. Pathogenesis of atherosclerosis. J Am Coll Cardiol 2006;47:C7-12.

8. Bergheanu SC, Bodde MC, Jukema JW. Pathophysiology and treatment of atherosclerosis. Current view and future perspective on lipoprotein modification treatment. Neth Heart J 2017;25:231-42.

9. Aziz M, Yadav K. Pathogenesis of Atherosclerosis A Review. Med Clin Rev 2016;2:3.

10. Standish BA, Spears J, Marotta TR, et al. Vascular Wall Imaging of Vulnerable Atherosclerotic Carotid Plaques: Current State of the Art and Potential Future of
Endovascular Optical Coherence Tomography. AJNR Am J Neuroradiol 2012;33:1642-50.

11. Singh RB, Mengi SA, Xu YJ, et al. Pathogenesis of atherosclerosis: A multifactorial process. Exp Clin Cardiol 2002; 7:40-53.

12. North American Symptomatic Carotid Endarterectomy Trial Collaborators, Barnett HJM, Taylor DW, et al. Beneficial effect of carotid endarterectomy in symptomatic patients with high-grade carotid stenosis. N Engl J Med 1991;325:445-53.

13. Barnett HJ, Taylor DW, Eliasziw M, et al. North American Symptomatic Carotid Endarterectomy Trial Collaborators. Benefit of carotid endarterectomy in patients with symptomatic moderate or severe stenosis. N Engl J Med 1998;339:1415-25.

14. European Carotid Surgery Trialists' Collaborative Group. MCR European Carotid Surgery Trial: interim results for symptomatic patients with severe (70-99\%) or with mild (0-29\%) carotid stenosis. Lancet 1991;337:1235-43.

15. European Carotid Surgery Trialists' Collaborative Group. Randomised trial of endarterectomy for recently symptomatic carotid stenosis: final results of the MRC European Carotid Surgery Trial (ECST). Lancet 1998;351:1379-87.

16. Saba L, Anzidei M, Marincola BC, et al. Imaging of the Carotid Artery Vulnerable Plaque. Cardiovasc Intervent Radiol 2014;37:572-85.

17. Rodriguez K, Kwan AC, Lai S, et al. Coronary Plaque Burden at Coronary CT Angiography in Asymptomatic Men and Women. Radiology 2015;277:73-80.

18. Gao T, Zhang Z, Yu W, et al. Atherosclerotic Carotid Vulnerable Plaque and Subsequent Stroke: A HighResolution MRI Study. Cerebrovascular Diseases 2009;27:345-52.

19. Coutinho JM, Derkatch S, Potvin ARJ, et al. Nonstenotic carotid plaque on CT angiography in patients with cryptogenic stroke. Neurology 2016;87:665-72.

20. Sun J, Zhao X-Q, Balu N, et al. Carotid Plaque Lipid Content and Fibrous Cap Status Predict Systemic CV Outcomes. JACC Cardiovasc Imaging 2017;10:241-9.

21. Ihdayhid AR, Goeller M, Dey D, et al. Comparison of Coronary Atherosclerotic Plaque Burden and Composition as Assessed on Coronary Computed Tomography Angiography in East Asian and European-Origin Caucasians. Am J Cardiol 2019;124:1012-9.

22. Bentzon JF, Otsuka F, Virmani R, et al. Mechanisms of Plaque Formation and Rupture. Circulation Research 2014;114:1852-66. 
23. Burke AP, Kolodgie FD, Farb A, et al. Morphological predictors of arterial remodeling in coronary atherosclerosis. Circulation 2002;105:297-303.

24. Daghem M, Bing R, Fayad ZA, et al. Noninvasive Imaging to Assess Atherosclerotic Plaque Composition and Disease Activity: Coronary and Carotid Applications. JACC Cardiovasc Imaging 2020;13:1055-68.

25. Crouse JR 3rd, Craven TE, Hagaman AP, et al. Association of coronary disease with segment-specific intimal-medial thickening of the extracranial carotid artery. Circulation 1995;92:1141-7.

26. Hollander M, Hak AE, Koudstaal PJ, et al. Comparison Between Measures of Atherosclerosis and Risk of Stroke The Rotterdam Study. Stroke 2003;34:2367-72.

27. Störk S, van den Beld AW, von Schacky C, et al. Carotid artery plaque burden, stiffness, and mortality risk in elderly men: a prospective, population-based cohort study. Circulation 2004;110:344-8.

28. Spence JD. Measurement of intima-media thickness vs. carotid plaque: uses in patient care, genetic research and evaluation of new therapies. Int J Stroke 2006;1:216-21.

29. Reilly LM, Lusby RJ, Hughes L, et al. Carotid plaque histology using real-time ultrasonography. Clinical and therapeutic implications. Am J Surg 1983;146:188-93.

30. Carotid artery plaque composition-relationship to clinical presentation and ultrasound b-mode imaging. Eur J Vasc Endovasc Surg 1995;10:23-30.

31. Coli S, Magnoni M, Sangiorgi G, et al. Contrast-enhanced ultrasound imaging of intraplaque neovascularization in carotid arteries correlation with histology and plaque echogenicity. J Am Coll Cardiol 2008;52:223-30.

32. Shah F, Balan P, Weinberg M, et al. Contrast-enhanced ultrasound imaging of atherosclerotic carotid plaque neovascularisation: A new surrogate marker of atherosclerosis? Vasc Med 2007;12:291-7.

33. Giannoni MF, Vicenzini E, Citone M, et al. Contrast carotid ultrasound for the detection of unstable plaques with neoangiogenesis: A pilot study. Eur J Vasc Endovasc Surg 2009;37:722-7.

34. Huang R, Abdelmoneim SS, Ball CA, et al. Detection of carotid atherosclerotic plaque neovascularization using contrast enhanced ultrasound: a systematic review and meta-analysis of diagnostic accuracy studies. J Am Soc Echocardiogr 2016;29:491-502.

35. Li Z, Bai Y, Li W, et al. Carotid vulnerable plaques are associated with circulating leukocytes in acute ischemic stroke patients: an clinical study based on contrastenhanced ultrasound. Sci Rep 2018;8:8849.
36. López-Melgar B, Fernández-Friera L, Oliva B, et al. Subclinical Atherosclerosis Burden by 3D Ultrasound in Mid-Life: The PESA Study. J Am Coll Cardiol 2017;70:301-13.

37. Spence JD. Approaching Automated 3-Dimensional Measurement of Atherosclerotic Plaque Volume. J Am Coll Cardiol 2017;70:314-7.

38. Mughal MM, Khan MK, DeMarco JK, et al. Symptomatic and asymptomatic carotid artery plaque. Expert Rev Cardiovasc Ther 2011;9:1315-30.

39. Serfaty JM, Chaabane L, Tabib A, et al. Atherosclerotic plaques: classification and characterization with T2weighted high-spatial-resolution MR imaging — an in vitro study. Radiology 2001;219:403-10.

40. Ruehm SG, Corot C, Vogt P, et al. Magnetic resonance imaging of atherosclerotic plaque with ultrasmall superparamagnetic particles of iron oxide in hyperlipidemic rabbits. Circulation 2001;103:415-22.

41. Sannino A, Brevetti L, Giugliano G, et al. Noninvasive vulnerable plaque imaging: how do we know that treatment works? Eur Heart J Cardiovasc Imaging 2014;15:1194-202.

42. Cocker MS, Spence JD, Hammond R. [18F]fluorodeoxyglucose PET/CT imaging as a marker of carotid plaque inflammation: Comparison to immunohistology and relationship to acuity of events. Int J Cardiol 2018;271:378-86.

43. Hop H, de Boer SA, Reijrink M, et al. 18F-sodium fluoride positron emission tomography assessed microcalcifications in culprit and non-culprit human carotid plaques. J Nucl Cardiol 2019;26:1064-75.

44. Cocker MS, Spence JD, Hammond R, et al. [18F]-NaF PET/CT Identifies Active Calcification in Carotid Plaque. JACC Cardiovasc Imaging 2017;10:486-8.

45. Skagen K, Skjelland M, Zamani M, et al. Unstable carotid artery plaque: new insights and controversies in diagnostics and treatment. Croat Med J 2016;57:311-20.

46. Hingwala D, Kesavadas C, Sylaja PN, et al. Multimodality imaging of carotid atherosclerotic plaque: Going beyond stenosis. Indian J Radiol Imaging 2013;23:26-34.

47. Saba L, Saam T, Jäger HR, et al. Imaging biomarkers of vulnerable carotid plaques for stroke risk prediction and their potential clinical implications. Lancet Neurol 2019;18:559-72.

48. Saba L, Biswas M, Kuppili V, et al. The present and future of deep learning in radiology. Eur J Radiol 2019;114:14-24.

49. Hermus L, Lefrandt JD, Tio RA, et al. Carotid plaque 
formation and serum biomarkers. Atherosclerosis 2010;213:21-9.

50. Saba L, Francone M, Bassareo PP, et al. CT Attenuation Analysis of Carotid Intraplaque Hemorrhage. AJNR Am J Neuroradiol 2018;39:131-7.

51. Wintermark M, Jawadi SS, Rapp JH, et al. HighResolution CT Imaging of Carotid Artery Atherosclerotic Plaques. AJNR Am J Neuroradiol 2008;29:875-82.

52. de Weert TT, de Monyé C, Meijering E, et al. Assessment of atherosclerotic carotid plaque volume with multidetector computed tomography angiography. Int J Cardiovasc Imaging 2008;24:751-9.

53. Saba L, Micheletti G, Brinjikji W, et al. Carotid Intraplaque-Hemorrhage Volume and Its Association with Cerebrovascular Events. AJNR Am J Neuroradiol 2019;40:1731-7.

54. Zhu G, Li Y, Ding V, et al. Semiautomated characterization of carotid artery plaque features from computed tomography angiography to predict atherosclerotic cardiovascular disease risk score. J Comput Assist Tomogr 2019. [Epub ahead of print].

55. Chrencik MT, Khan AA, Luther L, et al. Quantitative assessment of carotid plaque morphology (geometry and tissue composition) using computed tomography angiography. J Vasc Surg 2019;70:858-68.

56. Gupta A, Baradaran H, Schweitzer AD, et al. Carotid plaque MRI and stroke risk: a systematic review and metaanalysis. Stroke 2013;44:3071-7.

57. Sheahan M, Ma X, Paik D, et al. Atherosclerotic Plaque Tissue: Noninvasive Quantitative Assessment of Characteristics with Software-aided Measurements from Conventional CT Angiography. Radiology 2018;286:622-31.

58. Demarco JK, Ota H, Underhill HR, et al. MR carotid plaque imaging and contrast-enhanced MR angiography identifies lesions associated with recent ipsilateral thromboembolic symptoms: an in vivo study at 3T. AJNR Am J Neuroradiol 2010;31:1395-402.

59. Mitsumori LM, Hatsukami TS, Ferguson MS, et al. In vivo accuracy of multisequence $M R$ imaging for identifying unstable fibrous caps in advanced human carotid plaques. J Magn Reson Imaging 2003;17:410-20.

60. Saba L, Mallarini G. Fissured fibrous cap of vulnerable carotid plaques and symptomaticity: are they correlated? Preliminary results by using multi-detector-row CT angiography. Cerebrovasc Dis 2009;27:322-7.

61. Saba L, Tamponi E, Raz E, et al. Correlation between Fissured Fibrous Cap and Contrast Enhancement:
Preliminary Results with the Use of CTA and Histologic Validation. AJNR Am J Neuroradiol 2014;35:754-9.

62. Teng Z, He J, Degnan AJ, et al. Critical mechanical conditions around neovessels in carotid atherosclerotic plaque may promote intraplaque hemorrhage. Atherosclerosis 2012;223:321-6.

63. Saba L, Lai ML, Montisci R, et al. Association between carotid plaque enhancement shown by multidetector CT angiography and histologically validated microvessel density. Eur Radiol 2012;22:2237-45.

64. Zhao X, Hippe DS, Li R, et al. Prevalence and characteristics of carotid artery high-risk atherosclerotic plaques in Chinese patients with cerebrovascular symptoms: a Chinese Atherosclerosis Risk Evaluation II Study. J Am Heart Assoc 2017;6:e005831.

65. Saba L, Sanfilippo R, Pascalis L, et al. Carotid artery wall thickness and ischemic symptoms: evaluation using multi-detector-row CT angiography. Eur Radiol 2008;18:1962-71.

66. DeMarco JK, Huston J. Imaging of high-risk carotid artery plaques: current status and future directions. Neurosurg Focus 2014;36:E1.

67. Singh N, Moody AR, Panzov V, et al. Carotid intraplaque hemorrhage in patients with embolic stroke of undetermined source. J Stroke Cerebrovasc Dis 2018;27:1956-9.

68. Saam T, Hetterich H, Hoffmann V, et al. Meta-analysis and systematic review of the predictive value of carotid plaque hemorrhage on cerebrovascular events by magnetic resonance imaging. J Am Coll Cardiol 2013;62:1081-91.

69. Trelles M, Eberhardt KM, Buchholz M, et al. CTA for screening of complicated atherosclerotic carotid plaqueAmerican Heart Association type VI lesions as defined by MRI. AJNR Am J Neuroradiol 2013;34:2331-7.

70. Gupta A, Baradaran H, Kamel H, et al. Evaluation of computed tomography angiography plaque thickness measurements in high-grade carotid artery stenosis. Stroke 2014:45:740-5.

71. Rafailidis V, Chryssogonidis I, Tegos T, et al. Imaging of the ulcerated carotid atherosclerotic plaque: a review of the literature. Insights Imaging 2017;8:213-25.

72. Saba L, Sanfilippo R, Sannia S, et al. Association between carotid artery plaque volume, composition, and ulceration: a retrospective assessment with MDCT. AJR Am J Roentgenol 2012;199:151-6.

73. van Gils MJ, Homburg PJ, Rozie S, et al. Evolution of atherosclerotic carotid plaque morphology: do ulcerated plaques heal? A serial multidetector CT angiography 
study. Cerebrovasc Dis 2011;31:263-70.

74. de Weert TT, Cretier S, Groen H, et al. Atherosclerotic plaque surface morphology in the carotid bifurcation assessed with multidetector computed tomography angiography. Stroke 2009;40:1334-40.

75. Saba L, Caddeo G, Sanfilippo R, et al. Efficacy and sensitivity of axial scans and different reconstruction methods in the study of the ulcerated carotid plaque using multidetector-row CT angiography: comparison with surgical results. AJNR Am J Neuroradiol 2007;28:716-23.

76. Saba L, Caddeo G, Sanfilippo R, et al. CT and ultrasound in the study of ulcerated carotid plaque compared with surgical results: potentialities and advantages of multidetector row CT angiography. AJNR Am J Neuroradiol 2007;28:1061-6.

77. Yuan J, Usman A, Das T, et al. Imaging Carotid Atherosclerosis Plaque Ulceration: Comparison of Advanced Imaging Modalities and Recent Developments. AJNR Am J Neuroradiol 2017;38:664-71.

78. Wannarong T, Parraga G, Buchanan D, et al. Progression of carotid plaque volume predicts cardiovascular events. Stroke 2013;44:1859-65.

79. Ukwatta E, Yuan J, Rajchl M, et al. 3-D carotid multi-region MRI segmentation by globally optimal evolution of coupled surfaces. IEEE Trans Med Imaging 2013;32:770-85.

80. Saba L, Montisci R, Raz E, Sanfilippo R, Suri JS, Piga M. Association between carotid artery plaque type and cerebral microbleeds. AJNR Am J Neuroradiol 2012;33:2144-50.

81. Tarkin JM, Dweck MR, Evans NR, et al. Imaging Atherosclerosis. Circ Res 2016;118:750-69.

82. Mannelli L, Mitsumori LM, Ferguson M, et al. Changes in measured size of atherosclerotic plaque calcifications in dual-energy CT of ex vivo carotid endarterectomy specimens: effect of monochromatic $\mathrm{keV}$ image reconstructions. Eur Radiol 2013;23:367-74.

Cite this article as: Murgia A, Erta M, Suri JS, Gupta A, Wintermark M, Saba L. CT imaging features of carotid artery plaque vulnerability. Ann Transl Med 2020;8(19):1261. doi: 10.21037/atm-2020-cass-13
83. Saba L, Argiolas GM, Siotto P, et al. Carotid artery plaque characterization using CT multienergy imaging. AJNR Am J Neuroradiol 2013;34:855-9.

84. Shah PK. Can Carotid Plaque Predict Coronary Plaque?. JACC Cardiovasc Imaging 2013;6:1168-71.

85. Gupta A, Mushlin AI, Kamel H, et al. Cost-effectiveness of carotid plaque MR imaging as a stroke risk stratification tool in asymptomatic carotid artery stenosis. Radiology 2015;277:763-72.

86. Ochi N, Tabara Y, Igase $M$, et al. Silent cerebral microbleeds associated with arterial stiffness in an apparently healthy subject. Hypertens Res 2009;32:255-60.

87. Saba L, Sanfilippo R, di Martino M, et al. Volumetric Analysis of Carotid Plaque Components and Cerebral Microbleeds: A Correlative Study. J Stroke Cerebrovasc Dis 2017;26:552-8.

88. Uehara M, Funabashi N, Mikami Y, et al. Quantitative effect of atorvastatin on size and content of non-calcified plaques of coronary arteries 1 year after atorvastatin treatment by multislice computed tomography. Int J Cardiol 2008;130:269-75.

89. Soeda T, Uemura S, Okayama S, et al. Intensive LipidLowering Therapy With Rosuvastatin Stabilizes LipidRich Coronary Plaques. Circ J 2011;75:2621-7.

90. Mannelli L, MacDonald L, Mancini M, et al. Dual energy computed tomography quantification of carotid plaques calcification: comparison between monochromatic and polychromatic energies with pathology correlation. Eur Radiol 2015;25:1238-46.

91. Naim C, Douziech M, Therasse E, et al. Vulnerable atherosclerotic carotid plaque evaluation by ultrasound, computed tomography angiography, and magnetic resonance imaging: an overview. Can Assoc Radiol J 2014;65:275-86.

92. Vanninen R, Manninen H, Soimakallio S. Imaging of carotid artery stenosis: clinical efficacy and costeffectiveness. AJNR Am J Neuroradiol 1995;16:1875-83. 